Revista de Dialectología y Tradiciones Populares, vol. LXXI, n. ${ }^{\circ}$ 1, pp. 67-73, enero-junio 2016, ISSN: 0034-7981, eISSN: 1988-8457, doi: 10.3989/rdtp.2016.01.001.06

\title{
Experimentaciones participantes en arte y antropología
}

\section{Participatory Experimentation in Art and Anthropology}

\author{
Roger Sansi \\ Universitat de Barcelona \\ rogersansi@gmail.com
}

\section{RESUMEN}

En las páginas que siguen voy a proponer una reflexión sobre los dos conceptos fundamentales que guían esta sección: lo experimental y la colaboración. Me aproximaré a estos conceptos de forma consecutiva, primero el uno y después el otro, desde un punto de vista diferente al propuesto en la introducción: a partir del arte. Desde este ángulo, propongo algunas consideraciones más generales sobre las colaboraciones experimentales en antropología.

Palabras clave: Arte; Colaboración; Participación; Experimentación.

\section{SUMMARY}

The following article reflects on the two fundamental concepts dealt with in this section: the experimental and the collaborative dimensions. I shall approach each of these in turn, resorting, however, to a different perspective from the one proposed in the introduction, namely through art. From this angle, I shall offer more general considerations about experimental collaboration in anthropology.

Key words: Art; Collaboration; Participation; Experimentation.

\section{LO EXPERIMENTAL}

Lo experimental, como se dice en la introducción a esta sección, es una figura recurrente en el imaginario contemporáneo. Los autores de la introducción lo sitúan en una genealogía específica, en los ámbitos de la ciencia y la tecnología. Hay otra 
genealogía posible de lo experimental, que nos lleva hacia el arte de vanguardia. El término "experimental" se difunde a partir de los años cincuenta, en particular en relación con la música. En el ámbito de la música, lo experimental aparece con el uso de nuevas tecnologías de producción, grabación y reproducción. Pero el uso de estas tecnologías no se da tanto como un mecanismo de perfeccionamiento técnico - de control sobre el sonido- sino todo lo contrario: como una forma de liberación del sonido respecto a las intenciones del músico, de tal modo que la "música", se hace a sí misma como John Cage diría, "indeterminada", como resultado del azar. En ese sentido el uso de nuevas tecnologías se acompaña del uso de sonidos "no musicales" de la vida cotidiana, y de la creación de situaciones, en los cuales el espacio y el tiempo jugarían también el papel de "autores" de la música. El caso emblemático seria la pieza "4,33' de Cage: una composición que parte del silencio de los instrumentos para proponer escuchar el sonido ambiente.

Para Cage, una acción experimental seria aquella de la cual no se pueden prevenir sus resultados, una acción necesariamente única ${ }^{1}$. Esta definición parece muy diferente a las definiciones clásicas de la experimentación científica, que se basarían precisamente en lo opuesto: generar hipótesis que se puedan comprobar a través de métodos reproducibles en cualquier laboratorio con el equipamiento adecuado, siguiendo los protocolos establecidos.

En las últimas décadas, como muestra la introducción a esta sección, los estudios de ciencia y tecnología han cuestionado esta definición clásica, proveniente más de la filosofía de la ciencia que de la práctica científica en sí misma, que es y siempre ha sido múltiple. La ciencia crea sus propios objetos epistémicos, como dice (Rheinberger 1997); la práctica científica no es ajena a lo social, sino que hace parte de una "Cosmopolítica" (Stengers 2010), en la cual hay actantes humanos y no-humanos, de hecho, los actantes son resultado de estos mismos procesos experimentales (Latour 2001), que se conciben en términos de acontecimientos, no solo como protocolos reproducibles.

En el arte experimental estas cuestiones siempre has sido explícitas. El arte de vanguardia, desde el dadaísmo y el surrealismo, se concibe como una práctica de abandono de la intención artística en manos de las cosas, un encuentro y una inmersión en el mundo circundante en general ("la vida cotidiana"). El arte de vanguardia se constituye a través de diversos procesos que rehúyen del control técnico: el readymade, el automatismo, el objet trouvé, el cadáver exquisito.... Hasta llegar a los procesos experimentales de la música contemporánea. Procesos guiados por lo que Breton (1937) llamaría el azar objetivo, una correspondencia entre las series de causalidad naturales y sociales, donde objetos y sujetos se confunden. Esta noción del azar objetivo (por azar, por supuesto), coincide en el tiempo con la monografía sobre la magia Zande de Evans-Pritchard (1937), el cual describió la magia como un proceso de correspondencia entre acontecimientos humanos y naturales, en los cuales una coincidencia aparentemente azarosa se desvela como necesaria.

Podríamos entender la magia del etnógrafo en sí misma, a partir del principio del azar objetivo. Como Stocking (1992) arguyó, la influencia y el carisma del trabajo de

\footnotetext{
${ }^{1}$ "Una acción experimental es aquella cuyo resultado no se puede prever" (...) "necesariamente única" (Cage, 1961:39).
} 
campo etnográfico propuesto por Malinowski derivan más de la magia del etnógrafo que de una metodología explícita. A pesar de proponer enfáticamente el método etnográfico como "la aplicación de una serie de reglas del sentido común y bien conocidos principios científicos" de hecho, el trabajo de campo de Malinowski no era tanto una cuestión de métodos y recetas específicas, como de ponerse a uno mismo en una situación donde pudiese tener un cierto tipo de experiencia. Como la magia descrita por Malinowski en las Trobriand, el trabajo de campo era inicialmente imprevisible, y los elementos accidentales y de suerte muchas veces determinaban el éxito o el fracaso de la empresa (Stocking 1992: 58-9).

La magia del etnógrafo consistiría precisamente en poner estos retazos de experiencia del trabajo de campo en una narrativa coherente: la etnografía en sí, la monografía escrita. Este es el modelo sobre el cual se han educado generaciones de antropólogos: el trabajo de campo como un encuentro solitario con lo imprevisible, a partir del cual saldría (¿casi mágicamente?) una monografía. La contingencia (Battaglia 1999), los descubrimientos inesperados (Rivoal y Salazar 2013), adaptarse a lo desconocido, se consideran de hecho uno de sus mayores logros (Strathern 2000). El trabajo de campo sería un modo de investigación libre, abierto y auto-generado (Strathern 2000), y por lo tanto, muy difícil de reproducir, y de enseñar. El trabajo de campo es un acontecimiento siempre único e irrepetible. En ese sentido, no se encuentra tan lejos de la acción experimental de la que habla Cage. De hecho, la famosa y grandilocuente frase de Malinowski: "imagínate solo en una isla remota", parece una invocación surrealista al reencuentro con el mundo "allá afuera".

\section{LA COLABORACIÓN}

Muchos han notado la conexión entre el arte de vanguardias, en particular el surrealismo, y la antropología (Clifford 1988, Marcus y Myers 1995; Sansi 2015). Marcus ha señalado que la escena del encuentro malinowskiana no solo define un método particular, sino una cierta estética del trabajo de campo, un imaginario a partir del cual los antropólogos han constituido una imagen de sí (Marcus 2010: 266). Sin embargo, esta estética para Marcus debería ser re-imaginada, puesto que el contexto del trabajo de campo antropológico ha cambiado. En la actualidad, los antropólogos se encuentran con un mundo en el que el choque radical del exotismo y la alteridad, el mundo colonial de Malinowski, ha dado lugar a un "mundo en común", resultado de la globalización. En este contexto, la etnografía inevitablemente se transforma. Primero, el campo se vuelve múltiple, los "campos" son más difíciles de determinar, ya que no están aislados, sino conectados con otros lugares. También los sujetos etnográficos se mueven entre diferentes lugares, viajan y emigran, y muchas veces su estatus social y simbólico en este contexto global no es inferior al etnógrafo, sino igual o superior.

Este campo no es solo múltiple, sino que también está en proceso. El «mundo en común" no es un contexto establecido, con unos códigos bien definidos, sino que está siendo compuesto a partir de los diferentes agentes que intervienen en él (Latour 2015). Parte de esta intervención es similar al proceso etnográfico: muchos actores sociales producen formas de conocimiento afines a la etnografía, proponen definiciones de este campo emergente. El antropólogo no es el único que tiene autoridad para hablar en 
nombre de los otros. ¿Cuál es entonces la posición del antropólogo en este contexto en proceso? Para Marcus, el campo ya no es solo un "sitio" establecido, sino un "parasitio" (Marcus 2000), en construcción, resultado del trabajo en común de diferentes agentes, entre los cuales se encuentra el antropólogo. Estos otros agentes también están contribuyendo al proceso de construcción del campo, son para-etnógrafos, usando el prefijo "para", que define a un colaborador en un proceso de trabajo que en principio no es un experto, como por ejemplo, un para-médico. La etnografía, dice Marcus, necesita de los para-etnógrafos, si tiene que ser un proceso colaborativo.

A este punto nos podríamos preguntar si realmente, esta nueva estética de la colaboración que propone Marcus, está tan alejada del trabajo de campo clásico. Es cierto que hay diferencias claras de imaginario, diferencias estéticas, entre el modelo heroico de Malinowski, el investigador solitario que se adentra en las tinieblas del otro, y el modelo colaborativo, de proximidad, mucho más prosaico y realista, que propone Marcus. Pero más allá de la magia del etnógrafo de Malinowski, ¿Hasta qué punto las cuestiones que está destacando Marcus no han estado siempre presentes en el trabajo de campo? Tanto la multiplicidad e indeterminación del campo mismo y de los sujetos etnográficos, como la inevitable colaboración, que cuestiona los límites entre etnógrafo y etnografiado. Quizá por mucho tiempo, los etnógrafos dejaron de lado estas ambigüedades, puesto que el trabajo de campo etnográfico era la base de su legitimidad profesional, lo que constituía su marca de identidad y distinción. Sin embargo, multiplicidad y colaboración han hecho siempre parte del proceso.

\section{PARTICIPACIÓN}

Por otro lado, me parece importante que nos detengamos un momento a pensar qué es lo que entendemos por colaboración. Para hacerlo, me gustaría pensar este concepto en relación con otra palabra que a veces parece la misma: participación, y también en relación a otra disciplina afín, que produce algunas de estos paraetnografías: el arte contemporáneo.

En efecto, el arte continúa siendo un referente importante para entender los cambios en la estética del trabajo de campo, tal como los define Marcus. Si la estética del encuentro del trabajo de campo malinowskiano se encuentra en sintonía con el surrealismo y el arte de vanguardia, la estética de la colaboración de Marcus parece tener gran afinidad con las prácticas colaborativas y participativas que abundan en el arte contemporáneo.

Estas prácticas participativas o colaborativas se definen como procesos de trabajo que incluyen colectivos más allá del artista o el autor singular. Expandiendo el giro etnográfico que ya intuyó Hal Foster en los años noventa (1995), estas prácticas no son solo etnográficas por que les interesa el discurso de la diferencia, sino que se plantean como etnográficas en su metodología y voluntad de producir un conocimiento situado en un contexto especifico. Este conocimiento situado además se plantea en términos de una práctica política, no se plantea solo representar ciertos contextos sociales, sino intervenir sobre ellos y transformarlos.

Pero ¿de qué estamos hablando, cuando hablamos de participación o colaboración en arte? Este es sin duda un tema polémico. Muchas críticas se han levantado en los 
últimos años contra la "pesadilla de la participación" (Miesen 2011). La crítica a la participación sigue un argumento foucaultiano, por el cual proyectos de empoderamiento bien intencionados acaban convirtiéndose en dispositivos de disciplina y control, instrumentales en la construcción de subjetividades neoliberales (Bishop 2012). En reacción a estas críticas, Grant Kester (2011) defiende un arte colaborativo que define en términos del proceso de "trabajar juntos" (co-labour) en un modo de producción radicalmente opuesto a la explotación capitalista.

En este punto, haría falta considerar el significado de los términos de forma más amplia. "Colaboración" tiene un componente claro de horizontalidad, trabajar junto con, y responde bien a la ideología implícita que está por detrás de muchos proyectos, tanto en el arte como fuera de él: el individualismo igualitarista, que parte del principio que la colaboración es una decisión individual, voluntaria, de trabajar en un proyecto colectivo. No olvidemos que el "colaboracionismo" es una acusación dirigida a aquellos que trabajan con el enemigo por su propia voluntad, no forzados. El colectivo de colaboradores se define como un conjunto de individuos que pre-existen al colectivo y deciden formarlo. La separación ontológica entre individuos dados y colectivos construidos es una premisa implícita, que a pesar de la pátina post-estructuralista, está presente en muchos proyectos.

El término participación, por otro lado, puede implicar mucho más que un proceso colaborativo entre individuos. La participación es compartir, pero también hacer parte de, y compartirse. Para Lévy-Bruhl, en la "participación mística" el sujeto no se podía distinguir del objeto. Para Mauss, no había nada de místico, ni de misterioso, en la "participación": era por el contrario, una de las características principales de la problemática cotidiana, que propone el concepto de "don": "En el don, nos damos a nosotros mismos", decía Mauss. "Se mezclan las almas en las cosas y las cosas en las almas. Se mezclan las vidas y así es como las personas y las cosas mezcladas salen cada una de su esfera y se mezclan". (Mauss 1971: 91). En los dones, las cosas no son propiedad de las personas, sino parte de ellas $-\mathrm{y}$ al contrario. En el mismo sentido, dos personas pueden "mezclarse" y ser algo en común, a un nivel más profundo que una colaboración, para participar uno de otro. Esta pegajosidad del don es uno de los elementos sobre los que más han insistido las relecturas contemporáneas del don, en particular Marilyn Strathern cuando ha hablado de la persona "partible" o "distribuida" (1988). La noción de que la "persona" no se limita a un cuerpo y a una mente, sino que puede estar constituida por diferentes cuerpos y cosas, la persona no tiene porque ser un individuo/cuerpo sino que puede ser un conjunto de cuerpos y cosas.

Este "participar" el uno del otro, las cosas de las personas, los colectivos en los individuos y viceversa, no está en absoluto basado en una premisa de la igualdad. Esta es una cuestión que puede ser importante por ejemplo para repensar las prácticas artísticas participativas o colaborativas, que muchas veces se basan en intercambios de dones entre participantes que se conciben como igualitarios y voluntarios. Por el contrario, la literatura antropológica sobre el don ha dejado siempre claro que los intercambios de dones pueden ser jerárquicos y obligatorios Y esta jerarquía y obligación no solo representa relaciones entre personas sociales si no que las constituye, es decir, las personas son resultado de relaciones sociales, no su premisa. Este principio de la antropología relacional no es tan explicito, en cambio, en la "estética relacional" (Bourriaud 1998) de las prácticas artísticas contemporáneas. 
Esta visión más radical de la participación puede ser útil, de hecho, para entender el componente experimental de las prácticas artísticas participativas. Si el arte de vanguardia se concibe como una práctica de abandono de la intención artística en manos de las cosas, un encuentro y una inmersión en el mundo circundante, el arte contemporáneo participativo solo estaría llevando este principio a su consecuencia lógica, abandonando la intención artística no solo en manos de las cosas que el artista encuentra, sino del mundo social que lo constituye. Las performances, los happenings, las situaciones del arte contemporáneo serían una extensión experimental del readymade y los objets trouvés de sus predecesores.

\section{CONCLUSIÓN: LA EXPERIMENTACIÓN PARTICIPANTE}

Para concluir brevemente, y para seguir con el juego de palabras, ¿por qué no hablar de experimentación participante? Mi intención no es proponer todavía una tercera vía o modelo, sino hacernos reflexionar un poco más a fondo sobre los términos que usamos. La crítica a la "observación participante" parece centrarse más en la primera parte, "observación", con su connotación de distancia objetivante, que en la segunda parte "participante". La nueva estética colaborativa propuesta por Marcus quizá no tenga tanto a criticar a una "experimentación participante". De hecho, el trabajo de campo antropológico siempre ha sido un proceso de experimentar junto con otros; reducir el trabajo de campo a la observación es muy limitante. Además, pensar en términos de participación puede llevarnos un poco más allá de la educada "colaboración", que se plantea en términos de un igualitarismo voluntarista, una democracia ciudadana ilustrada. La antropología puede aportar una visión más radical, de procesos participativos en los que no se trata solo de trabajar juntos por el bien común, sino de hacerse y deshacerse los unos a los otros. Esta visión radical no es necesariamente nueva, quizá ha estado siempre implícita en el trabajo de campo etnográfico. Pero siempre va bien repensar la etnografía a partir de otros modelos — como las colaboraciones experimentales-.

\section{BIBLIOGRAFÍA CITADA}

Battaglia, Deborah. 1999. "Towards an Ethics of the Open Subject: Writing Culture in Good Conscience", en Henrietta Moore (ed.), Anthropological Theory Today: 111-150. Cambridge: Polity Press.

Bishop, Claire. 2012. Artificial Hells: Participatory Art and the Politics of Spectatorship. Londres y Nueva York: Verso.

Bourriaud, Nicolas. 1998. Esthétique relationnelle. Dijon: Les Presses du réel.

Breton, André. 1937. L'amour fou. Paris: Gallimard.

Cage, John. 1961. Silence: Lectures and Writings. Middleton, Con.: Wesleyan U. P.

Clifford, James. 1988. The Predicament of Culture. Twentieth Century Ethnography, Literature, and Art. Cambridge, Mass.: Harvard University Press.

Evans-Pritchard, Eduard E. 1937. Witchcraft, Oracles, and Magic among the Azande. Oxford: Oxford U.P. Foster, Hal. 1995. "The Artist as Ethnographer?", en George E. Marcus y Fred Myer (eds.), The Traffic in Culture: Refiguring Art and Anthropology: 302-309. Berkeley: University of California Press.

Kester, Grant. 2011. The One and the Many: Contemporary Collaborative Art in a Global Context. Durham: Duke University Press. 
Latour, Bruno. 2001. La esperanza de Pandora. Ensayos sobre la realidad de los estudios de la ciencia. Barcelona: Gedisa.

Latour, Bruno. 2015. ¿El cosmos de quién?, ¿qué cosmopolítica? Commentarios sobre los términos de paz de Ulrich Beck". Pleyades 14: 43-39.

Lévy-Bruhl, Lucien. 1911. Les fonctions mentales dans les societies inferieures. Paris: Presses Universitaires de France.

Malinowski, Bronislaw. 1978 [1922]. Argonauts of the Western Pacific. Nueva York: Routledge.

Marcus, George E. 2000. Para-Sites: A Casebook Against Cynical Reason. Chicago: The University of Chicago Press.

Marcus, George E. 2010. "Contemporary Fieldwork Aesthetics in Art and Anthropology: Experiments in Collaboration and Intervention". Visual Anthropology 23: 263-277.

Marcus, George y Fred Myers (eds.) 1995. The Traffic in Culture. Berkeley: University of California Press.

Mauss, Marcel. 1971. Sociología y antropología. Taurus: Madrid.

Miessen, Marcus. 2011. The Nightmare of Participation. Berlin: Sternberg Press.

Rheinberger, Hans-Jörg. 1997. Towards a History of Epistemic Things. Stanford: Stanford University Press.

Rivoal, Isabelle y Noel B. Salazar. 2013. "Contemporary Ethnographic Practice and the Value of Serendipity". Social Anthropology 21(2): 178-185.

Sansi, Roger. 2015. Art, Anthropology and the Gift. Londres: Bloomsbury Publishing.

Stengers, Isabelle. 2010 Cosmopolitics I. Minneapolis: University of Minnesotta Press.

Stocking, George. 1992. The Ethnographer's Magic and Other Essays. Madison: University of Wisconsin Press.

Strathern, Marilyn. 1988. The Gender of the Gift. Berkeley: University of California Press.

Strathern, Marilyn (ed.). 2000. Audit Cultures: Anthropological Studies in Accountability, Ethics and the Academy. Londres y Nueva York: Routledge.

Fecha de recepción: 3 de marzo de 2016*

Fecha de aprobación: 9 de mayo de 2016

* Las fechas de recepción y aprobación vienen referidas al conjunto de la sección TEMAs EMERGENTES. 